CASE REPORT

\title{
A rare case of invasive thymoma presented a dramatic response to low-dose prednisolone as a single-drug therapy.
}

\author{
Masaki Hanibuchi', Atsuro Saijo ${ }^{1}$, Atsushi Mitsuhashi², Tatsuya Kajimoto' ${ }^{1}$, and Tetsuya Kitagawa ${ }^{3}$ \\ ${ }^{1}$ Department of Respiratory Medicine, ${ }^{3}$ Department of Cardiovascular Surgery, Shikoku Central Hospital of the Mutual aid Association of \\ Public School teachers, Shikoku-Chuo, Japan, ${ }^{2}$ Department of Respiratory Medicine and Rheumatology, Graduate School of Biomedical Sci- \\ ences, Tokushima University, Tokushima, Japan
}

\begin{abstract}
A 76-year-old woman with a history of angina pectoris, hypertension and dyslipidemia was pointed out an abnormal opacity in the right hilar region on routine chest X-ray. Chest computed tomography showed masses in the anterior mediastinum with the invasion of the adjacent ascending aorta, right brachiocephalic vein and right pleura. Histologic examination led to a diagnosis of Masaoka stage IVa thymoma. Three courses of chemotherapy were given, but further tumor progression was seen. Thereafter, the patient was followed without aggressive treatments. One year after the initial diagnosis, she presented with dyspnea and right chest pain. Chest CT revealed right massive pleural effusion with pleural dissemination and much further progression of existing tumors. For the purpose of symptom palliation, a low dose $(5 \mathrm{mg} / \mathrm{day})$ of prednisolone was commenced, which unexpectedly led to marked alleviation of patient's symptoms and dramatic decrease of pleural effusion. To the best of our knowledge, this is the first report of an invasive thymoma responded to low-dose corticosteroid. The present case suggests that corticosteroids, even at low doses, might be potentially effective for invasive thymoma after failure of surgery, chemotherapy and radiotherapy. J. Med. Invest. 68: 396-399, August, 2021
\end{abstract}

Keywords : invasive thymoma, low-dose corticosteroid, single-drug therapy

\section{INTRODUCTION} num, and arises from thymic epithelial cells with non-neoplastic T lymphocytes at varying degrees $(1,2)$. Surgical resection has been advocated as the primary treatment of thymoma, since it is often found well encapsulated $(3,4)$. In patients with advanced diseases or a recurrence after primary therapy, systemic chemotherapy can be considered (5). It has been reported that moderate to high doses of corticosteroids including steroid pulse therapy as a single-drug treatment also induced regression of thymoma (69). Kobayashi et al. demonstrated that the steroid pulse therapy induced tumor regression in 8 of 17 (47.1\%) patients with advanced thymoma and that the reduction in tumor size was most prominent in World Health Organization (WHO) classification, type B1 thymomas compared with type B3 (10). Although the pro-apoptotic effects are considered to play an important role in the growth-inhibiting action (11), the significance and the optimal dose of corticosteroid therapy in patients with thymoma is poorly understood.

In this report, we demonstrated a patient who had Masaoka clinical stage IVa invasive thymoma (WHO classification, type B3) complicated with massive pleural effusion and pleural dissemination, which remarkably responded to low-dose prednisolone (PSL).
Thymoma is the most common tumor of the anterior mediasti-

\section{CASE REPORT}

A 76-year-old woman with a history of angina pectoris, hypertension and dyslipidemia was pointed out an abnormal opacity in the right hilar region on routine chest X-ray. Chest computed tomography (CT) showed masses in the anterior mediastinum adjacent to the ascending aorta, right brachiocephalic vein and right pleura. These findings contraindicated curative surgery. A partial resection was performed and a diagnosis of Masaoka clinical stage IVa thymoma with the invasion of surrounding organs, such as right brachiocephalic vein, right internal thoracic vein, right mediastinum, right lung and pleura, was made. A histologic examination revealed that tumors were composed mainly of epithelial cells showing squamoid features and focal mild atypia with scant lymphocytes (WHO classification, type B3 thymoma). The positivity of Ki-67 was $20-30 \%$ in atypical epithelial cells. After diagnosis, three courses of chemotherapy (carboplatin and paclitaxel) were given, but a mass adjacent to the ascending aorta further enlarged. Although alternative chemotherapies were recommended, she refused the proposal. Thereafter, the patient was referred to our hospital for supportive care and palliative treatments of invasive thymoma six months after the initial diagnosis.

At the initial admission to our hospital, she did not complain of any respiratory symptoms and her general condition was relatively good. The physical examinations revealed body temperature of $36.3{ }^{\circ} \mathrm{C}$, blood pressure of $113 / 63 \mathrm{mmHg}$, pulse of 59 beats per minute and percutaneous oxygen saturation of $97 \%$ on room air. The remainder of the physical examinations was also

Received for publication May 14, 2021 ; accepted August 10, 2021.

Abbreviations

WHO, World Health Organization ; PSL, prednisolone ; CT, computed tomography ; GR, glucocorticoid receptor
Address correspondence and reprint requests to Masaki Hanibuchi, Department of Respiratory Medicine, Shikoku Central Hospital of the Mutual Aid Association of Public School teachers, 2233 Kawanoe-cho, Shikoku-Chuo, 799-0193, Japan and Fax : +81-896-58-3464. 
normal including chest auscultation. Muscle weakness was not recognized. The laboratory findings showed mild leukocytopenia and anemia. The serum level of human chorionic gonadotropin was within normal range and the acetylcholine receptor antibody was negative. The remainder of laboratory test results is shown in Table 1. We continued to follow the patient without aggressive treatments.

One year after the initial diagnosis, she presented with dyspnea and right chest pain. Chest CT revealed right massive pleural effusion and much further progression of existing tumors (Figure 1). Although the cytology of pleural effusion was negative (class II), we considered the etiology of pleural effusion as pleural

Table 1. Laboratory data at the first admission

\begin{tabular}{|c|c|c|c|c|c|}
\hline \multicolumn{2}{|l|}{ Hematology } & \multicolumn{4}{|c|}{ Biochemistry } \\
\hline WBC & $3,400 / \mu \mathrm{L}$ & AST & $25 \mathrm{IU} / \mathrm{L}$ & BS & $98 \mathrm{mg} / \mathrm{dL}$ \\
\hline $\mathrm{Neu}$ & $62.8 \%$ & ALT & $16 \mathrm{IU} / \mathrm{L}$ & HbA1c (NGSP) & $5.8 \%$ \\
\hline Lymph & $29.5 \%$ & ALP & $192 \mathrm{U} / \mathrm{L}$ & $\mathrm{BNP}$ & $26.3 \mathrm{pg} / \mathrm{mL}$ \\
\hline Mono & $5.9 \%$ & $\mathrm{LDH}$ & $159 \mathrm{IU} / \mathrm{L}$ & $\mathrm{PT}$ & 11.8 secs \\
\hline Eos & $1.2 \%$ & $\gamma$-GTP & $27 \mathrm{U} / \mathrm{L}$ & PT activity & $100 \%$ \\
\hline Baso & $0.6 \%$ & $\mathrm{CK}$ & $87 \mathrm{U} / \mathrm{L}$ & APTT & 27.8 secs \\
\hline $\mathrm{RBC}$ & $324 \times 10^{4} / \mu \mathrm{L}$ & T-bil & $0.5 \mathrm{mg} / \mathrm{dL}$ & CEA & $1.3 \mathrm{ng} / \mathrm{mL}$ \\
\hline $\mathrm{Hb}$ & $11.1 \mathrm{~g} / \mathrm{dL}$ & $\mathrm{TP}$ & $7.5 \mathrm{~g} / \mathrm{dL}$ & CYFRA 21-1 & $3.4 \mathrm{ng} / \mathrm{mL}$ \\
\hline $\mathrm{Ht}$ & $32.3 \%$ & $\mathrm{Alb}$ & $4.2 \mathrm{~g} / \mathrm{dL}$ & hCG & $4.4 \mathrm{mIU} / \mathrm{mL}$ \\
\hline \multirow[t]{9}{*}{ Plt } & $17.6 \times 10^{4} / \mu \mathrm{L}$ & T-cho & $130 \mathrm{mg} / \mathrm{dL}$ & Anti-Ach-R Ab & $<0.2 \mathrm{nmol} / \mathrm{L}$ \\
\hline & & Amy & $115 \mathrm{IU} / \mathrm{L}$ & & \\
\hline & & BUN & $16.3 \mathrm{mg} / \mathrm{dL}$ & & \\
\hline & & Cre & $0.53 \mathrm{mg} / \mathrm{dL}$ & & \\
\hline & & $\mathrm{Na}$ & $143 \mathrm{mEq} / \mathrm{L}$ & & \\
\hline & & K & $3.9 \mathrm{mEq} / \mathrm{L}$ & & \\
\hline & & $\mathrm{Cl}$ & $107 \mathrm{mEq} / \mathrm{L}$ & & \\
\hline & & $\mathrm{Ca}$ & $9.6 \mathrm{mg} / \mathrm{dL}$ & & \\
\hline & & $\mathrm{CRP}$ & $0.05 \mathrm{mg} / \mathrm{dL}$ & & \\
\hline
\end{tabular}

BNP, brain natriuretic peptide ; CEA, carcinoembryonic antigen ; CYFRA 21-1, cytokeratin fraction 21-1; hCG, human chorionic gonadotropin ; Anti-Ach-R Ab, anti-acetylcholine receptor antibody

A

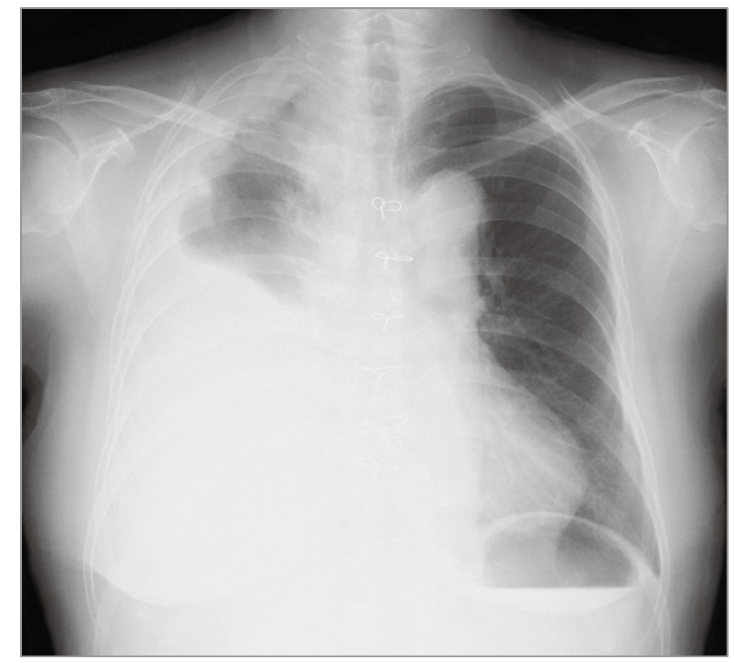

B

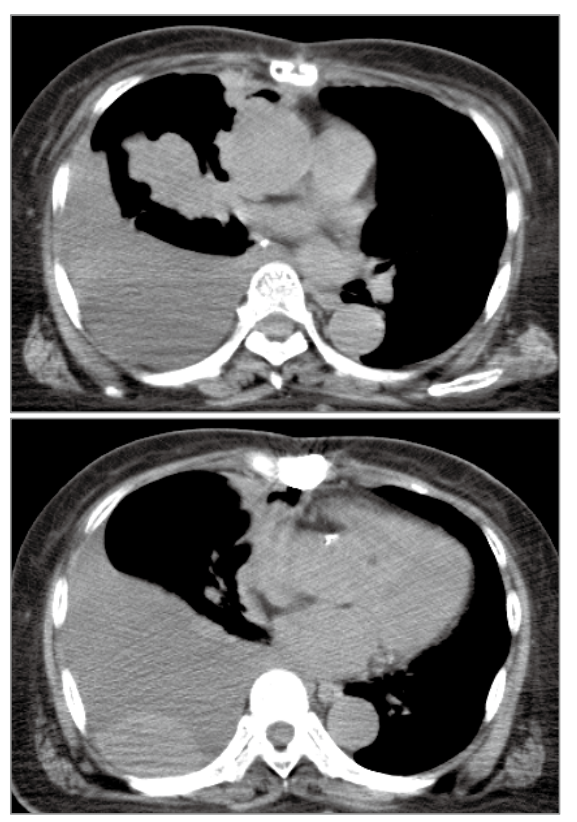

Figure 1. Chest X-ray and Chest CT findings before corticosteroid treatment.

Right massive pleural effusion and mass shadow in the right upper mediastinum was evident in chest X-ray (Figure 1A). Chest CT revealed right massive pleural effusion and tumors in the right hilum, anterior mediastinum, and right pleura (Figure 1B). 
dissemination of invasive thymoma. Thoracentesis, and palliative radiation therapy to the right hilum, apex and chest wall temporarily alleviated her symptoms. Thoracic cavity drainage by the chest drainage tube and pleurodesis were not performed following the patient's wishes. Even after that, thoracenteses were performed as appropriate and supportive treatment for bilateral pretibial and foot-pad edema, such as anti-diuretics, were commenced, however, pleural effusion gradually increased and her symptoms, such as dyspnea and general fatigue got worse. For symptom palliation, we started a low dose ( $5 \mathrm{mg} /$ day) of PSL. As her body weight was $46.7 \mathrm{~kg}$, the dose of PSL was approximately $0.1 \mathrm{mg} / \mathrm{kg} /$ day at the treatment initiation. Unexpectedly, her symptoms markedly improved and pleural effusion dramatically decreased on chest X-ray at three-weeks after corticosteroid initiation. Chest CT also showed significant reduction of right pleural effusion and regression of tumors in the right hilum and pleura (Figure 2). The general condition and right pleural effusion of the patient remain well-controlled even now while continuing to receive $4 \mathrm{mg} /$ day of PSL at six-months after corticosteroid initiation. No adverse events have been reported during treatment, with the exception of mild insomnia.

\section{DISCUSSION}

Corticosteroids are known to induce regression of thymoma, whereas these are often administered to patients with thymoma to treat associated symptoms of myasthenia gravis (6) and pure red-cell aplasia (12) rather than the tumor itself. There has been a small number of single case reports of response to corticosteroids alone in patients with thymoma, mostly after failure of combined chemoradiotherapy and surgery (6-8). In these reports, moderate to high doses of corticosteroids including steroid pulse therapy (more than $30 \mathrm{mg} /$ day of PSL) were administered, however, there is no report describing therapeutic efficacy of low-dose corticosteroid in patients with invasive thymoma. To the best of our knowledge, this is the first report of an invasive thymoma presented a dramatic response to low-dose corticosteroid as a single-drug therapy.

Since the first description of regression of thymoma by the treatment of adrenocorticotropic hormone (13), several reports have indicated that corticosteroids might be potentially effective for invasive thymoma $(7,14)$, primarily in cases of lymphocytic thymoma, and have been attributed to the apoptotic effects of corticosteroids, mainly on the lymphocytic component of the tumor $(8,12)$. On the other hand, glucocorticoid receptor (GR) is shown to be expressed on neoplastic thymic epithelial cells as well as on non-neoplastic thymocytes and lymphocytes in thymoma. Glucocorticoids induced apoptosis via G1 cell cycle arrest of thymic epithelial cells, indicating that glucocorticoids directly suppressed in vitro proliferation of GR-positive neoplastic thymic epithelial cells (15). In addition, studies to evaluate histopathologic changes of thymoma preoperatively treated with high doses of corticosteroids, such as steroid pulse therapy, demonstrated apoptosis induction in neoplastic thymic epithelial cells, as well as lymphocytes in the specimens resected after corticosteroid treatment $(9,10)$. These findings suggested that corticosteroids cause degenerative changes in neoplastic thymic epithelial cells, as well as lymphocytes in thymoma.

The association of therapeutic efficacies of corticosteroid with histological subtypes of thymoma remains controversial. Kobayashi et al. demonstrated that the steroid pulse therapy induced tumor regression in patients with advanced thymoma and that the tumor reduction was most prominent in type B1 thymoma (also known as lymphocytic thymoma) compared with type B3 thymoma (also known as epithelial thymoma) (10). Moreover, glucocorticoids directly suppressed the proliferation of GR-positive neoplastic thymic epithelial cells via the induction of apoptosis (15), and the numbers of GR sites were reported to be much greater in lymphoepithelial thymoma as compared to those in pure epithelial thymoma (16). These observations support a hypothesis that lymphocytic component of thymoma
A

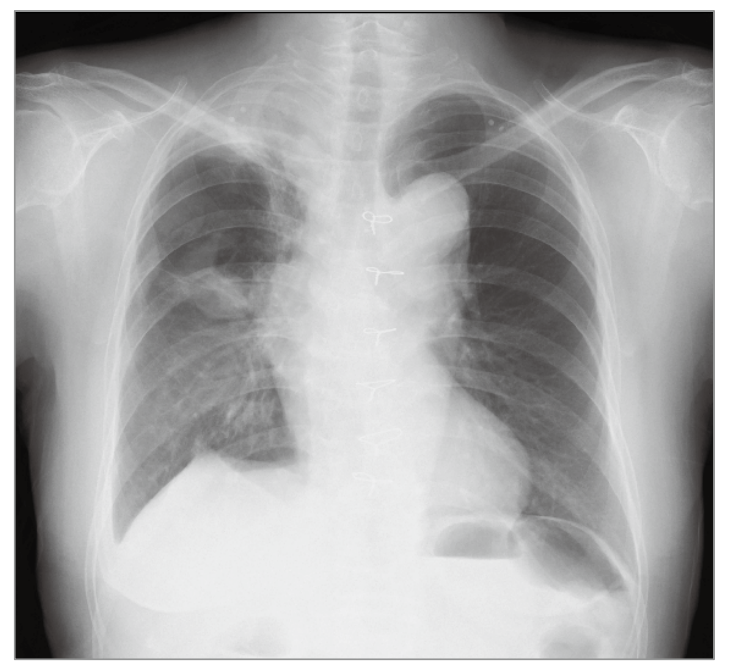

B

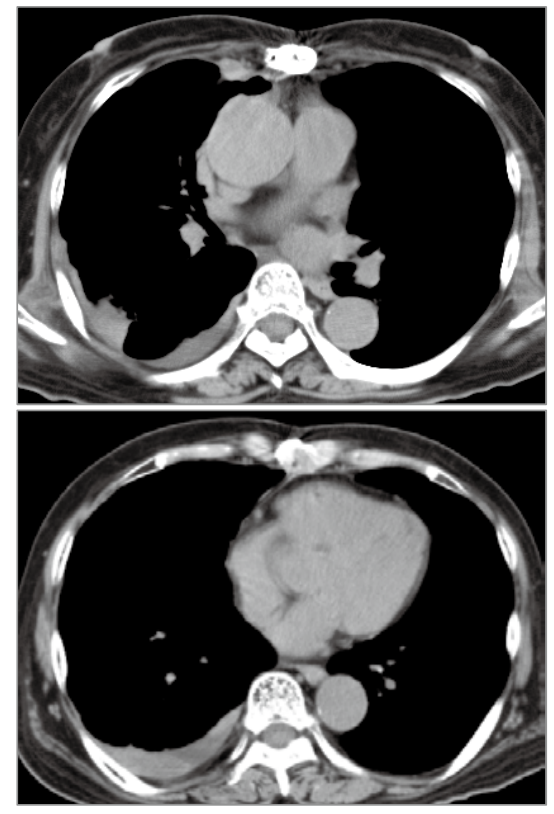

Figure 2. Chest X-ray and Chest CT at six-months after corticosteroid initiation.

Right pleural effusion dramatically decreased and the shrinkage of tumor in the right upper mediastinum also observed in chest X-ray (Figure 2A). Chest CT showed significant reduction of right pleural effusion and regression of tumors in the right hilum and pleura (Figure 2B). 
is more responsive to corticosteroid treatment. At the other extreme, Hu et al. reported that glucocorticoid therapy induced thymoma regression in 11 of 13 cases, regardless of different histologic findings (17). In addition, it is shown that high doses of corticosteroids, such as steroid pulse therapy, induced apoptosis of neoplastic thymic epithelial cells, as well as lymphocytes in thymoma (9). In the present case, although we could not evaluate the pathological change after treatment, corticosteroid therapy, even at low doses, might affect not only lymphocytic components but also neoplastic thymic epithelial cells in WHO classification, type B3 thymoma.

In terms of the prognostic markers for thymoma, the histological subtype is considered to be a promising candidate. However, previous study to examine the histopathologic features of pretreatment biopsies and posttreatment surgical specimens of thymoma demonstrated the significant changes of the histological subtypes after treatment including corticosteroids (9). As the conversion of histological subtype might affect the therapeutic response and the clinical course, it is possible that the pretreatment histopathologic features do not necessarily reflect the prognosis of thymoma. Recently, Mimae et al. reported that GR revealed a high rate of expression (82.9\%) in thymomas and thymic carcinomas. Multivariate analysis demonstrated that GR expression was associated with better prognosis in patients with surgically resected thymomas and thymic carcinomas (18). Moreover, Kobayashi et al. showed that the reduction in tumor size after steroid pulse therapy was accompanied with a marked reduction in the CD4+8+ double-positive immature lymphocytes (10), and indicated that tumor size reduction of thymomas was partly explained by the reduction in the bulk of these lymphocytes. Thus, GR expression and CD4+8+ double-positive immature lymphocytes might be new promising candidates as prognostic markers for thymoma. However, abovementioned observations need to be interpreted cautiously, because the sample size in each study was quite limited. Further large-scale studies would be required to show the more clinically significant data in the future.

In conclusion, we herein reported a rare case of invasive thymoma presented a dramatic response to low-dose corticosteroid as a single-drug therapy. Five mg/day of PSL that used for the purpose of symptom palliation markedly alleviated patient's symptoms and pleural effusion dramatically decreased. To the best of our knowledge, this is the first report of an invasive thymoma responded to low-dose corticosteroid. The present case suggests that corticosteroids even at low doses, as well as those at high doses, might be potentially effective for invasive thymoma after failure of surgery, chemotherapy and radiotherapy.

\section{CONFLICT OF INTEREST DISCLOSURE}

All authors state that they do not have any financial or other relationships for the present study that might lead to a conflict of interest.

\section{ACKNOWLEDGEMENTS}

We thank our colleagues at Shikoku Central Hospital of the Mutual aid Association of Public School teachers, especially Takumi Uda and Hitomi Komoda for technical assistance.

\section{REFERENCES}

1. Levine GD, Rosai J : Thymic hyperplasia and neoplasia. A review of current concepts. Hum Pathol $9: 495-515,1978$

2. Verley J, Hollmann KH : Thymoma. A comparative study of clinical stages, histologic features, and survival in 200 cases. Cancer 55 : 1074-1086, 1985

3. Gripp S, Hilgers K, Wurm R, Schmitt G : Thymoma : prognostic factors and treatment outcomes. Cancer $83: 1495$ 1503,1998

4. Okumura M, Miyoshi S, Takeuchi Y, Yoon HE, Minami M, Takeda SI, Fujii Y, Nakahara K, Matsuda H : Results of surgical treatment of thymomas with special reference to the involved organs. J Thorac Cardiovasc Surg 117 : 605613,1999

5. Chahinian AP, Bhardwaj S, Meyer RJ, Jaffrey IS, Kirschner PA, Holland JF : Treatment of invasive or metastatic thymoma : report of eleven cases. Cancer $47: 1752-1761,1981$

6. Kodama K, Doi O, MD, Higashiyama M, Yokouchi H, Yasuda T, Funai H : Dramatic response of postthymomectomy myasthenia gravis with multiple lung nodules to corticosteroids. Ann Thorac Surg 64 : 555-557, 1997

7. Termeer A, Visser FJ, Mravunac M : Regression of invasive thymoma following corticosteroid therapy. Neth J Med $58: 181-184,2001$

8. Tiseo M, Monetti F, Ferrarini M, Serrano J, Chiaramondia $\mathrm{M}$, Aradizzoni A : Complete remission to corticosteroids in an octreotide-refractory thymoma. J Clin Oncol 23 : 15781579,2005

9. Tateyama H, Takahashi E, Saito Y, Fukai I, Fujii Y, Niwa H, Eimoto T : Histopathologic changes of thymoma preoperatively treated with corticosteroids. Virchows Arch 438 : 238-247, 2001

10. Kobayashi Y, Fujii Y, Yano M, Sasaki H, Yukiue H, Haneda H, Suzuki E, Endo K, Kawano O : Preoperative steroid pulse therapy for invasive thymoma : clinical experience and mechanism of action. Cancer $106: 1901-1907,2006$

11. Kruman II, Matylevich NP, Beletsky IP, Afanasyev VN, Umansky SR : Apoptosis of murine BW 5147 thymoma cells induced by dexamethasone and gamma irradiation. J Cell Physiol 148: 267-273, 1991

12. Palmieri G, Lastoria S, Colao A, Vergara E, Varrella P, Biondi E, Selleri C, Catalano L, Lombardi G, Bianco AR, Salvatore M : Successful treatment of a patient with thymoma and pure red-cell aplasia with octreotide and prednisone. New Engl J Med 336 : 263-265, 1997

13. Soffer LJ, Gabrilove JL, Wolf BS : Effect of ACTH on thymic masses. J Clin Endocrinol Metab 12 : 690-696, 1952

14. Kirkove C, Berghmans J, Noel H, van de Merckt J : Dramatic response of recurrent invasive thymoma to high doses of corticosteroids. Clin Oncol $4: 64-66,1992$

15. Funakoshi Y, Shiono H, Inoue M, Kadota Y, Ohta M, Matsuda H, Okumura M, Eimoto T: Glucocorticoids induce G1 cell cycle arrest in human neoplastic thymic epithelial cells. J Cancer Res Clin Oncol 131:314-322, 2005

16. Ranelletti FO, Iacobelli S, Carmignani M, Sica G, Natoli C, Tonali P : Glucocorticoid receptors and in vitro corticosensitivity in human thymoma. Cancer Res 40 : 2020-2025, 1980

17. $\mathrm{Hu} \mathrm{E}$, Levine $\mathrm{J}$ : Chemotherapy of malignant thymoma. Case report and review of the literature. Cancer $57: 1101$ 1104, 1986

18. Mimae T, Tsuta K, Takahashi F, Yoshida A, Kondo T, Murakami Y, Okada M, Takeuchi M, Asamura H, Tsuda $\mathrm{H}$ : Steroid receptor expression in thymomas and thymic carcinomas. Cancer 117 : 4396-4405, 2011 